\title{
Microfabricated tools for nanoscience
}

\author{
J Bruggert, V P Jaecklin, C Linder, N Blanc, P F Indermühle \\ and NF de Rooij
}

Institute of Microtechnology (IMT), University of Neuchâtel, Breguet 2, $\mathrm{CH}-2000$

Neuchâtel, Switzerland

\begin{abstract}
Recent developments and advances in micro-electro-mechanical systems for nanometer-scale applications such as scanning force microscopy are presented. The microfabrication of tools so small that they enable access to the nanoworld, such as tips, flexible cantilevers, integrated deflection sensors and nanoactuators will be described. Bulk and surface micromachining of monoor polycrystalline siljicon, extended by aligned-wafer-bonding, etch-back and sacrificial-layer-etching steps are major fabrication steps used. Experiments on prototypes show that these devices are promising for use as ultra-sensitive stand-alone local probe micro-instruments.
\end{abstract}

\section{Introduction}

The invention of the scanning tunneling microscope (STM) [1] and the atomic or scanning force microscope (AFM, SFM) [2] has opened completely new opportunities for high-resolution surface-probing instruments for research and industrial purposes [3]. Real-space atomicscale images of samples have been obtained and revolutionized the fields of surface physics, chemistry and biology. New generations of instruments for quality inspection of device surfaces, e.g. in ULSI integrated circuitry or for optical glasses became feasible. The expected number of sales of STMS/SFMS for 1993 is about eight hundred, representing a new market with an expected annual increase of 5-10\% [4].

In addition to the imaging mode these new instruments can be used for the controlled indentation of various surfaces for nanometer-scale recording [5]. Even the manipulation of individual atoms has been demonstrated [6], which might produce information storage devices with terabit storage densities for the 21st century [7].

Access to the nanoworld is typically gained by means of a local probe operating with surface contact or in a near-field mode at a few ångströms sample distance only. In the case of an SFM a tiny cantilever beam with a sharp tip is scanned across the surface, and the interaction between the probe and the surface atoms or topographical protrusions is monitored via the motion of the cantilever. Scanning in a raster mode enables the creation of real-space surface images. The control and monitoring of the position and interaction between the probe and the sample requires extremely small and accurate handling tools with tight specifications. In order to be able to measure small forces of nano- to picoNewton magnitude, a cantilever with low spring

† Present address: Advanced Research Laboratory, Hitachi Ltd, Kokubunji-shi Tokyo 185 Japan. constant is required. On the other hand high resonant frequencies are preferred to increase both the scan rates and the immunity of the device to external noise interference. These requirements lead naturally to a reduction in size of the entire device, which is therefore of extremely tiny mass with a low system compliance able to directly access and measure nanometer- and atomic-scale events. In order to transmit and amplify the small signals we need transducers, e.g. for obtaining feedback information from the surfaces during the imaging or manipulation process. A Nano-robot system' as macro-to-nano mediation has recently been presented [8]. Micromechanics in silicon [9] and complete micro-electro-mechanical systems (MEMSs) are most suitable as interfacing parts of the nine orders of magnitude in dimensions that separate our human world with its traditional mechanical instruments and the nanometer world. MEMS technology benefits from the large amount of expertise gained during the last three decades in microelectronics technologies using batch fabrication processes with low unit costs.

Intensive research activities have been launched worldwide for nanotechnology including the development of nano-instruments with a high degree of function integration. New generations of integrated siliconbased sensors and actuators for local probe systems have been presented over the past few years. Silicon micromachining is one key technology for the fabrication of such devices as outlined recently by MacDonald [10]. The scaling of the structure dimensions from the already 'classical' micrometer to the nanometer plays a dominant role, which lies beyond the limits of standard microfabrication methods. Further miniaturization may therefore require the improvement and extension of novel combinations of high-resolution lithographic (e-beam) and etching techniques $[10,11]$.

The spatial resolution of a local probe system (such as an SFM) is strongly related to the probe tip radius and the distance to the sample. Tip shape, sharpness, 
aspect ratio and material are essential and different types may be required for the various applications in mind (high-resolution images of flat samples, imaging of deep-structured surfaces, nanometrology, etc) [12]. Many ways of producing SFM tips have been described. Basically two methods are nowadays used to yield tiplike-structures. The first is anisotropic and/or isotropic etching of a single-crystal material such as silicon and subsequent tip sharpening by an oxidation process, thus exploiting natural effects as a self-sharpening procedure $[13,14]$. The other method is to use a focused beam for either the deposition of a contamination shaft in a scanning electron microscope [15] or, alternatively, to sharpen by ion milling an existing tip of various materials [16].

Pioneering results in the field of microfabricated SFM cantilevers with integrated tips have been obtained by means of various surface and/or bulk micromachining techniques $[17,18]$ and are meanwhile routinely used for mass production of these fragile replaceable structures. A novel simple single-mask fabrication process has been published recently [19]. Furthermore, as a trend to a higher degree of autonomous functionality, integrated piezoresistive [20] and piezoelectric cantilever deflection sensors [21] have been successfully implemented. As for the tip actuation, a microfabricated STM scan tip using a zinc oxide piezoelectric bimorph cantilever has been demonstrated [22]. In-plane electrostatic actuators for nanometer-precise positioning of a center stage have also been fabricated by surface micromachining [2326]. An overhanging microgripper has been operated [27]; such a device could facilitate the access of a local probe sensor to a flat sample surface. Also, novel displacement sensors using vacuum tunneling seem to be most promising devices for the highest sensitivity [28] and results for a micromachined high-sensitivity accelerometer based on this measurement principle have been presented [29].

For over a decade the group of de Rooij has been contributing to the advances of microfabricated sensors and actuators and their integration into microsystems. As part of the trend toward continuous miniaturization we aim for the complete integration of a micromachined nanosystem composed of an array of local probes, sensor elements and actuator mechanisms for the tip-versussample $x y$-scanning and for the tip-versus-sample $z$ control, i.e. its flight height. A challenging goal will be to obtain a really protruding cantilever/tip in order to solve the access problem to the specimen in real-life applications. Furthermore scanning the tip instead of the sample stage would make the system independent of sample size and a stand-alone microscanner could be obtained. Sensor elements for positioning and interaction measurements are required and should not interfere with the actuator parts.

In this paper we present an overview of microfabricated silicon tools fabricated in our laboratory which represent the basic components of an MEMS for nanoscience, in this particular case for an SFM. We show cantilever arrays with integral tips, an integrated

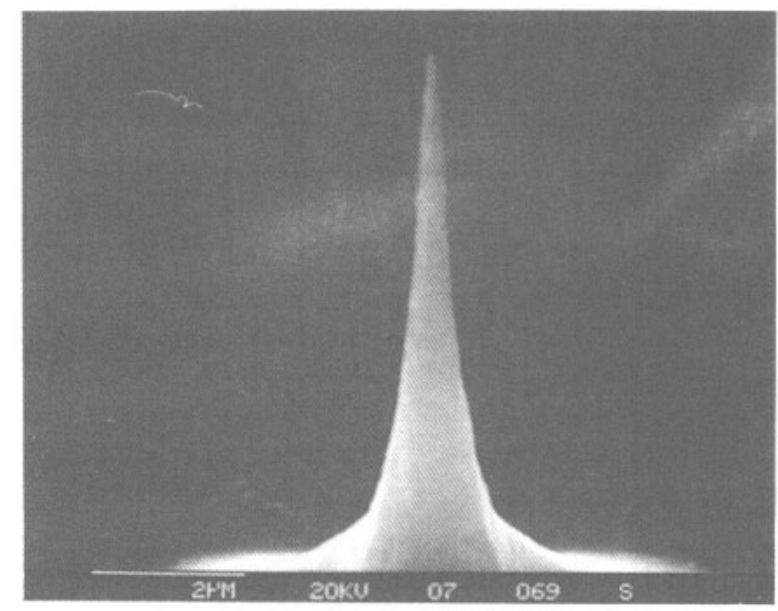

FIgure 1. SEM micrograph of an etched silicon tip with high aspect ratio and a tip radius of less than $50 \mathrm{~nm}$.

cantilever deflection sensor/actuator using a capacitive device and surface micromachined poly- and singlecrystal silicon $x y$ nanoactuators. The approach to a fully integrated scanned probe microsystem will be discussed.

\section{Tips}

A rather simple two-step process for making silicon tips has been developed. A photolithographic pattern (polygon or circle) of $1.5 \mu \mathrm{m}$ thick thermal silicon dioxide is used as an etch mask for reactive ion etching (RIE) using $\mathrm{SF}_{6} / \mathrm{C}_{2} \mathrm{ClF}_{5}$ gases to obtain a $10 \mu \mathrm{m}$ silicon column. The etch parameters are adjusted in order to control the undercut and the slope of the side walls [30]. The second step consists of an isotropic wetetching process in a mixture of $\mathrm{HNO}_{3}: \mathrm{HF}: \mathrm{CH}_{3}: \mathrm{COOH}$ where the silicon column is progressively thinned and finally ends in a sharp tip when the cap is completely underetched. By this method we achieved high-aspectratio tips with a height of more than $10 \mu \mathrm{m}$ and tip radii of less than $50 \mu \mathrm{m}$. By changing the etch parameters different shapes of tips can be tailored to some degree. Figure 1 shows the detailed view of a highly protruding silicon tip fabricated by the described procedure.

\section{Batch-fabricated SFM cantilevers with integrated tips}

A difficult task in SFM always has been the integration of a sharp protruding tip onto the tiny flexible cantilevered force sensor. Our group has developed a three-mask IC-compatible batch process using combined wet and dry etching. A more detailed description is given in previous publications, e.g. [31]. Bricfly, a thermally oxidized silicon wafer is double-side patterned: on the top side for the RIE etching of the cantilevertip structures and on the reverse side for the final membrane back etching. The oxide layer $(1.5 \mu \mathrm{m})$ on the top side is patterned by means of two subsequent photolithography and etch steps into a bi-level mask 


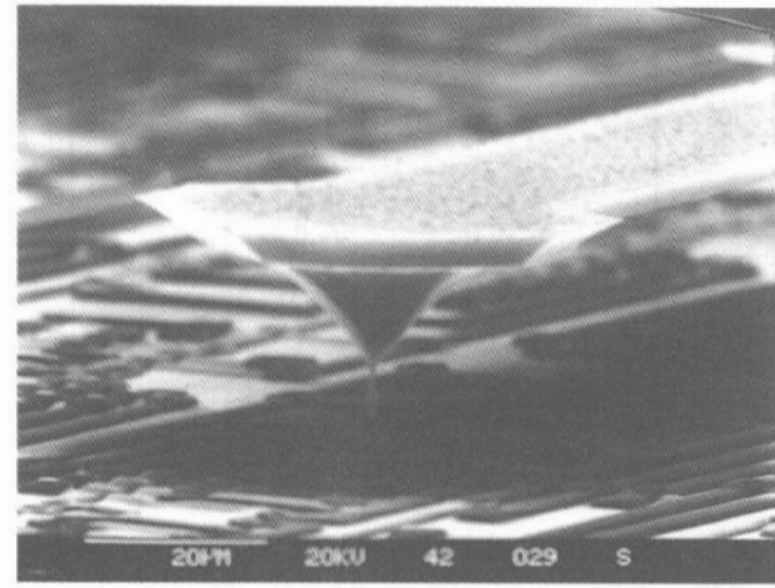

Figure 2 SEM micrograph of a microfabricated silicon SFM cantilever with the tip hovering over an integrated circuit surface.

layer with the tip layout keeping the whole oxide thickness and the cantilever layout reduced to half of it $(0.75 \mu \mathrm{m})$. This process variant allows for the patterning of both the cantilever and the tip on the flat substrate before continuing with the deep micromachining etching processes. The cantilever is machined using RIE parameters keeping the side walls rather vertical [30]. Afterwards the cantilever mask $(0.75 \mathrm{mum})$ is removed in buffered hydrofluoric acid (BHF) still leaving the additional $0.75 \mu \mathrm{m}$ oxide cap for the subsequent tip RIE step. The wafers are then time-controlled backetched in $40 \% \mathrm{KOH}$ solution at $60^{\circ} \mathrm{C}$ while the top side is protected in a mechanical chuck. Final wet etching in $\mathrm{HNO}_{3}: \mathrm{HF}: \mathrm{CH}_{3} \mathrm{COOH}$ finishes the tip-mask underetching, pierces through the membrane and thus releases the free-standing cantilevers with integrated tips, which are attached to a holder piece for mounting in an SFM system. By different pattern dimensions of the cantilevers, various mechanical specifications can be met: spring constants varying from 0.01 to $100 \mathrm{~N} \mathrm{~m}^{-1}$ and resonant frequencies up to $150 \mathrm{kHz}$ have been fabricated. Arrays of multiple probes have also been demonstrated (figure 3 ). The produced probes have been successfully used in commercial SFM systems, in both contact and non-contact imaging modes. Figure 4 shows an SFM topographic image of a section of a Fresnel phase lens etched in glass (Courtesy of $M$ Binggeli, CSEM Neuchâtel).

\section{Integrated SFM sensor/actuator (capacltive)}

The deflection of the tiny SFM cantilever induced by the forces during surface scanning can be monitored by different methods. Most common in commercial SFMS is the optical measurement of the beam deflection. Other displacement sensing such as vacuum tunneling, interferometric and capacitive detection have also been successfully implemented. These 'two-body' assembled set-ups require external components to be accurately aligned with the cantilever and isolated against thermal drift and vibration and complicate the instrument

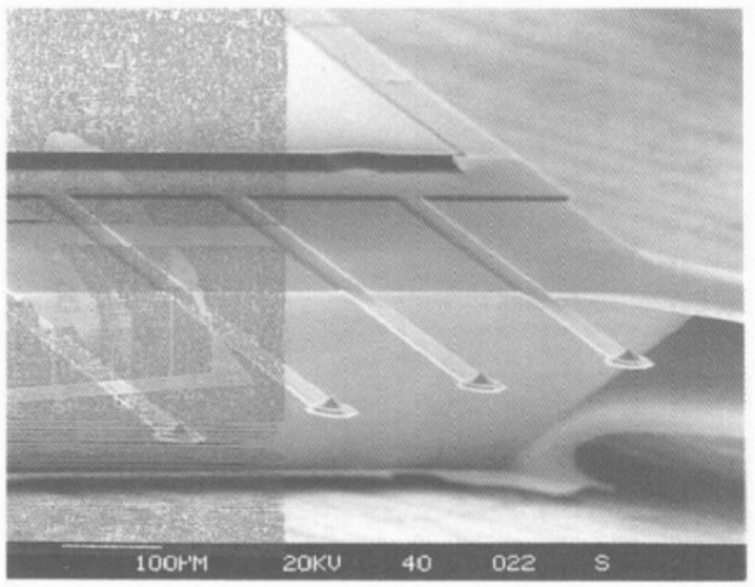

Figure 3. SEM micrograph of an array of four differentshaped silicon cantilevers with integrated tips.

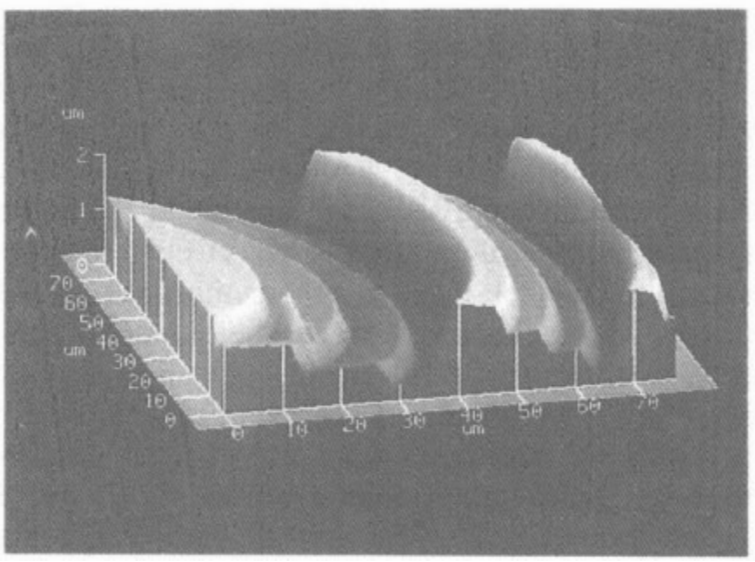

Figure 4. SFM topographic image of a section of a Fresnel phase lens etched in a glass (picture taken by $M$ Binggeli, CSEM Neuchâtel).

design. In consideration of an integrally designed SFM sensor head, a piezoresistive [20] and a piezoelectric [21] cantilever beam have been operated successfully. Such auto- or self-sensing probes are of great interest for miniaturized scanned probe devices since they enable the scanning of the tip versus the fixed sample while having the sensor incorporated in the probe, thus simplifying substantially the design of a standalone instrument. The use of a capacitor for the deflection measurement of an SFM cantilever has proven to be an interesting alternative to the other methods [32-35]. The fabrication and operation of a miniaturized capacitive probe is inherently critical. Strict instrumental requirements have to be ensured in order to control and minimize stray capacitance effects for high resolution.

We have developed such a novel SFM probe head consisting of a silicon microlever with tip and incorporated air-gap capacitor. The device is entirely fabricated by silicon micromachining as described in detail elsewhere [36]. Briefly, two silicon wafers are RIE processed separately to form cantilever and counter-electrode. Wafer alignment and pre-bonding within $2 \mu \mathrm{m}$ alignment accuracy is performed on a 


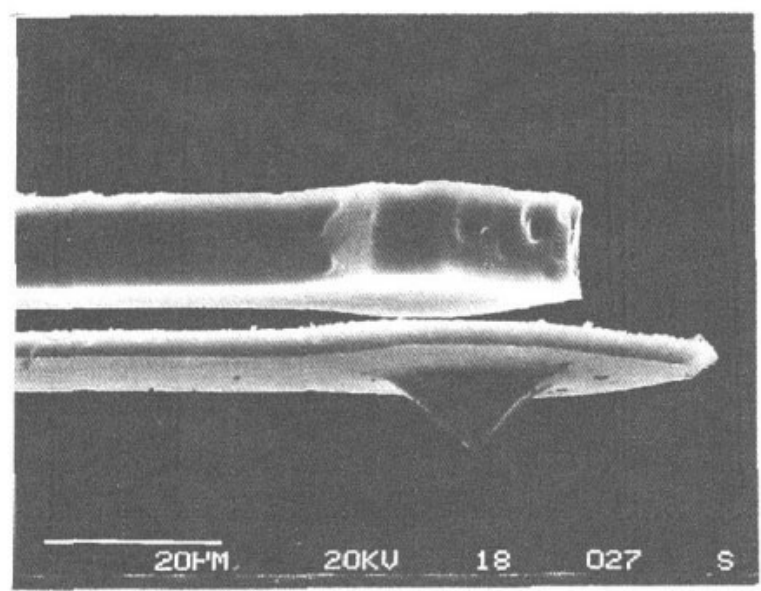

Figure 5. SEM micrograph of a micromachined silicon capacitive microlever with tip for SFM.

commercial bond aligner followed by fusion bonding at $1100^{\circ} \mathrm{C}$. Then the wafers are etched in $\mathrm{KOH}$ and a tip mask is patterned onto the membrane that covers the 'buried' cantilever precisely at the desired place over the cantilever head. Alignment marks on the wafer reverse side are used for this step. Subsequent RIE forms the tip column and final wet etching finishes the tips and pierces through the membranes. Finally, the air-gap capacitance between the silicon beams is opened by selective lateral etching of the intermediate bond oxide in BHF. Figure 5 shows an SEM micrograph of a capacitive microlever fabricated by the described procedure. Typical dimensions are $800 \times 40 \times 5 \mu \mathrm{m}^{3}$ for the microlever length, width and thickness respectively yielding values of spring constant $c=0.5 \mathrm{~N} \mathrm{~m}^{-1}$ and resonant frequency $f=10 \mathrm{kHz}$. The air gap is defined by the thickness of the bond oxide and was in our case $3 \mu \mathrm{m}$, which yields for the given dimensions a lever capacitance of $C=0.1 \mathrm{pF}$. The sensitivity of the device in terms of vertical tip displacement $\mathrm{d} C / \mathrm{d} z$ is estimated as $10 \mathrm{fF} \mu \mathrm{m}^{-1}$ which requires electronic circuitry for detection. The counter-electrode beam is considerably thicker than the cantilever beam and bending due to the small forces is assumed to be negligible, compared to the cantilever.

In addition to the deflection-sensing mode this capacitor device can also be used for $z$ actuation of the flexible cantilever with the integrated tip by applying controlled voltages between the two beams. Actuated tips are of great interest especially when probe arrays for parallel probing are considered. Then, each tip has to be individually addressable and movable in order to maintain the critical flight height over a possibly rough sample during the surface imaging or manipulation process. The described configuration can be used as tip-to-sample-distance fine adjustment. By the same mechanism the compliance of the local probe system can be modified. A theoretical calculation that takes into account the electrostatic force and the restoring spring force shows that by increasing the applied bias voltage the total potential energy of the deflected cantilever (i.e. the sum of the electrostatic energy and the strain energy)

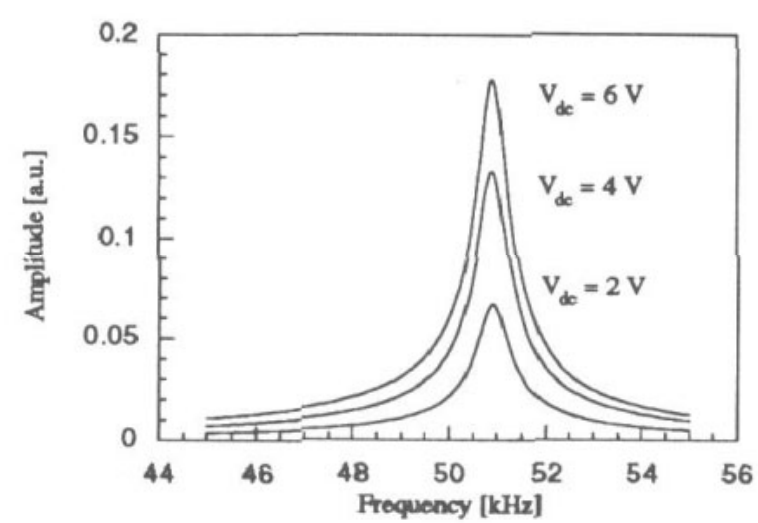

Figure 6. Frequency response of the actuated silicon microlever: resonant frequency, $51 \mathrm{kHz} ; V_{D C}=2,4,6 \mathrm{~V}$ i $V_{A C}=1 \mathrm{~V}$ peak to peak.

decreases [37]. At a certain threshold voltage the equilibrium position vanishes and the cantilever 'snaps' into the counter-electrode since beyond this threshold the electrostatic force is permanently larger than the restoring spring force in the whole displacement range. The described mechanism enables to some extent the tailoring of the contact pressure between tip and sample. This gives access to new fields of SFM applications and is particularly interesting for imaging of soft surfaces on biological species.

Another possible application of this device is to use it as the driving mechanism for cantilever oscillation [38]. Silicon is a well suited resonator material due to its inherently high $Q$ factor [39]. In this particular case the cantilever is vibrated near the resonant frequency as required for non-contact surface profiling or tapping. When brought into close proximity with a sample, force gradients acting on the tip change the frequency and amplitude of vibration, which can both be measured. A first demonstration of this driving mechanism is given in figure 6. The graph shows the frequency response of a typical capacitive microlever. The amplitude was monitored with a confocal optical microscope. At a resonant frequency of $51 \mathrm{kHz}$ an amplitude of $60 \mathrm{~nm}$ could be observed for a DC bias of $6 \mathrm{~V}$ and an $\mathrm{AC}$ voltage of $1 \mathrm{~V}$ (peak to peak).

\section{XY nanoactuator}

Recently, sacrificial-layer technology, has gathered much interest as a new method of constructing micromechanical elements using fabrication techniques of the IC industry $[40,41]$. Most devices based on this technology are driven electrostatically; in particular the comb actuator proved to be a useful driving element [25-27]. Its main features are quick response time, IC-compatible driving voltages, no hysteresis in the positioning and the feasibility of an integrated capacitive positioning detection. These features make the comb actuator an interesting candidate for the positioning of an SFM tip $[23,42]$.

In the following, nanostructures are discussed that use polysilicon (in the next section silicon is employed) 


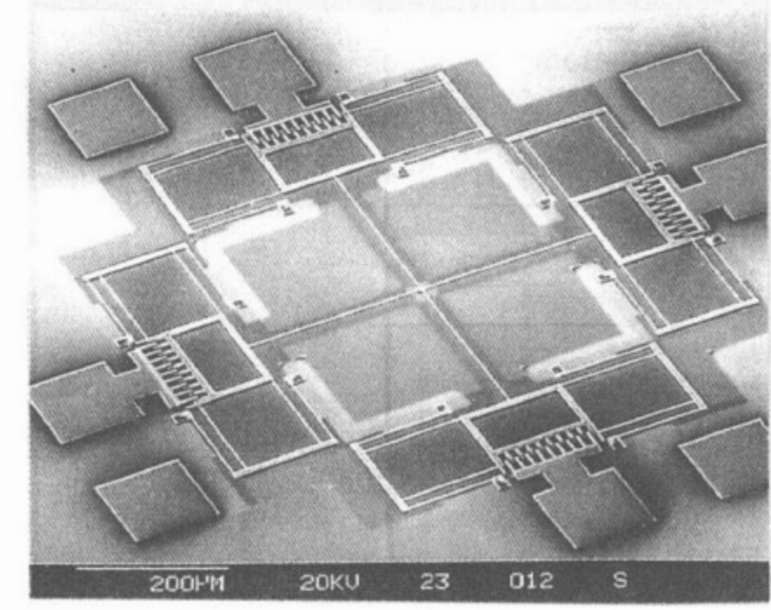

Flgure 7. View of a polysilicon comb-driven $x y$ nanoactuator.

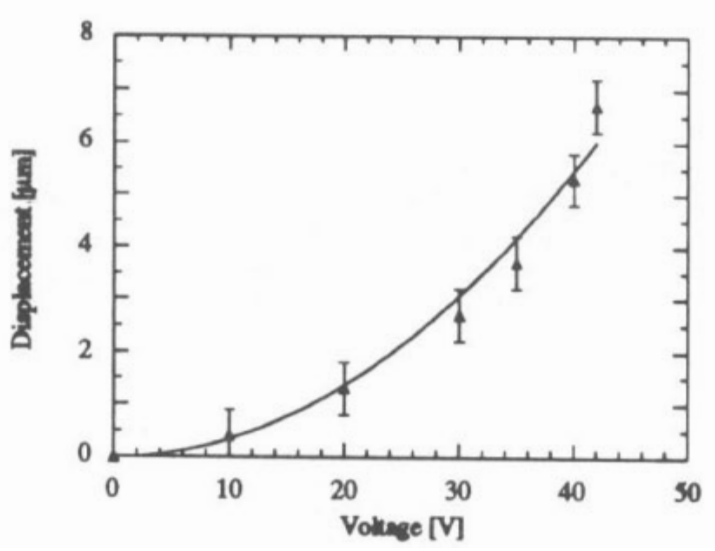

Figure B. Displacement-versus-voltage diagram of the polysilicon $x y$ nanoactuator.

as the structural layer and silicon dioxide as the sacrificial layer. A typical $x y$ nanopositioner that we fabricated in collaboration with the Swiss Center for Electronics and Microtechnology Inc (CSEM) in Neuchâtel, Switzerland, is shown in figure 7. As can be seen from the SEM micrograph the positioner consists of four comb actuators assembled around a center stage. The comb actuator itself consists of two electrodes, the fixed electrode and the movable electrode. The functional components of the latter are the suspension, which gives a mechanical guidance for the actuator movement, and the comb, where the force for the actuation is created. The design of the positioner is based on a pull mechanism-the table is moved to any position in the $x y$ plane by energizing two of the four comb drives. The decoupling of the $x$ and $y$ positions is given by the construction of the suspension. Figure 7 shows a complete view of the fabricated stage. The square table $(8 \mu \mathrm{m} \times 8 \mu \mathrm{m})$ is suspended by four $270 \mu \mathrm{m}$ long, $0.8 \mu \mathrm{m}$ wide and $2 \mu \mathrm{m}$ high beams.

With this prototype it was possible to displace the table in any direction in the $x y$ plane. Mechanically, a maximum surface of $30 \mu \mathrm{m} \times 30 \mu \mathrm{m}$ could be covered. For the electrostatic actuation a displacement-versusvoltage curve is shown in figure 8 . While the points are the measured displacements, the curve is drawn by an equation discussed in [42]. In particular, a $5.3 \mu \mathrm{m}$ displacement in the $x$ direction has been observed if on the corresponding comb actuator a voltage of $40 \mathrm{~V}$ is applied. FEM simulations of these structures showed that precise positioning can be performed because there is a good common mode rejection ratio of the displacements in the $x$ and $y$ directions. For the structure shown in figure 7 , the simulated ratio is more than $35 \mathrm{~dB}$. Hence, this device can be used for positioning in the $x y$ plane in the range of up to $10 \mu \mathrm{m}$ $\times 10 \mu \mathrm{m}$ with a precision of better than $50 \mathrm{~nm}$. The range could be confirmed in experiments using the light microscope and the SEM.

\section{Scanned probe mlcrosystem}

In order to integrate a sharp protruding tip onto a microfabricated $x y$-scan device, we investigated the feasibility of fabricating an $x y$ scanner out of singlecrystal silicon. A schematic view of the complete device is shown in figure 9. The fabrication is performed by means of advanced silicon micromachining and is similar to the process for the previously described capacitive microlever device. The actuated movable parts are released by selective etching of the sactificial dioxide under the structural beams. The process sequence features initial RE processing of two wafers and subsequent alignment and fusion bonding. After back etching of the silicon wafer bulk, an etch mask is patterned for RIE tip etching. Final wet etching of the silicon membrane and selective oxide etching releases the structural layer. Figure 10 is a micrograph of the realized device. The probe tip (8 $\mu \mathrm{m}$ high, estimated tip radius, $40 \mathrm{~nm}$ ) is integrated on the center stage of the $x y$ manipulator (figure 11). The most critical step is the etching of the back-side hole since stressinduced buckling of the thin membranes due to the oxicie layer tend to break the ultra-thin beams. Details of the processing are given elsewhere [43].

\section{Summary}

We have presented an overview of essential components designed for a surface-scanned local probe system such as the SFM. Advanced micromachining techniques are used to build tools so small that they enable access to nanometer dimensions. For instance, sharp tips and flexible cantilever force sensors have been routinely fabricated by combinations of wet and dry silicon etching and are used successfully in SFM systems. By using further micromachining steps such as aligned wafer bonding and sacrificial layer etching more complex devices have been constructed. In particular, an entirely micromachined SFM sensor/actuator based on capacitive microlever has been demonstrated. Theoretical modeling and first experiments show promising results for this device to be used as a highly sensitive local probe microinstrument. Surface micromachined $x y$ nanoactuators fabricated by poly-crystalline silicon have 


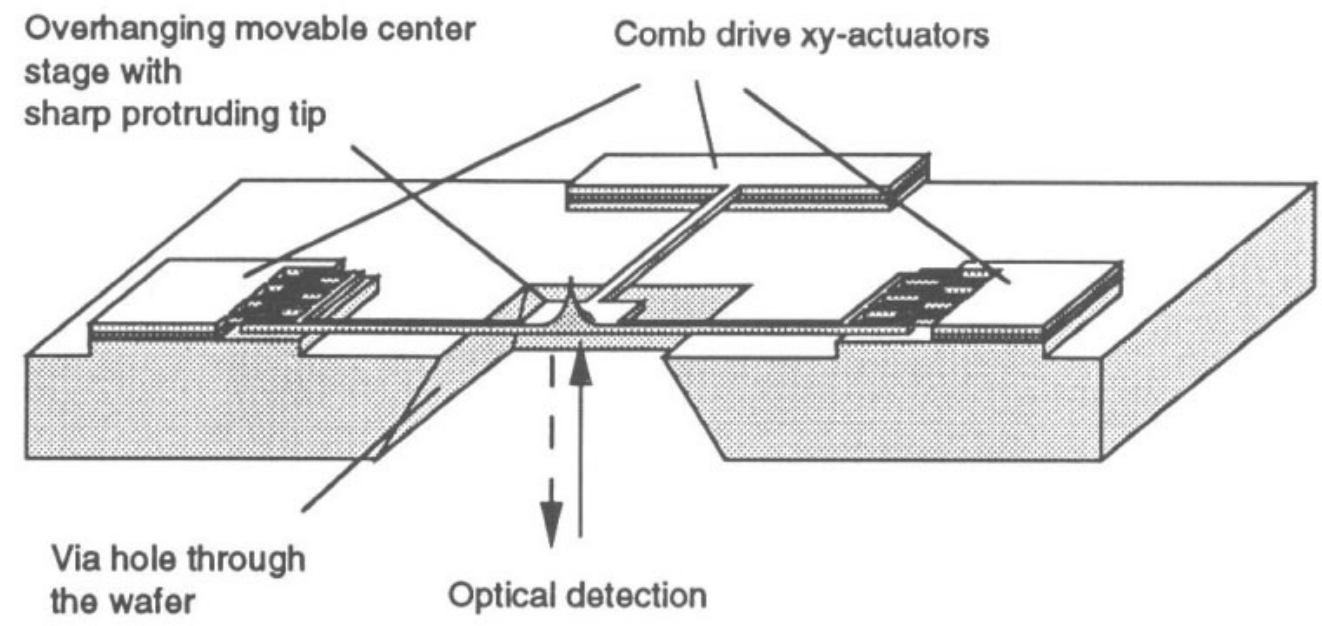

Figure 9. Schematic representation of the micromachined monocrystalline silicon $x y$ scanner with integrated tip and overhanging structural beams for backside monitoring.

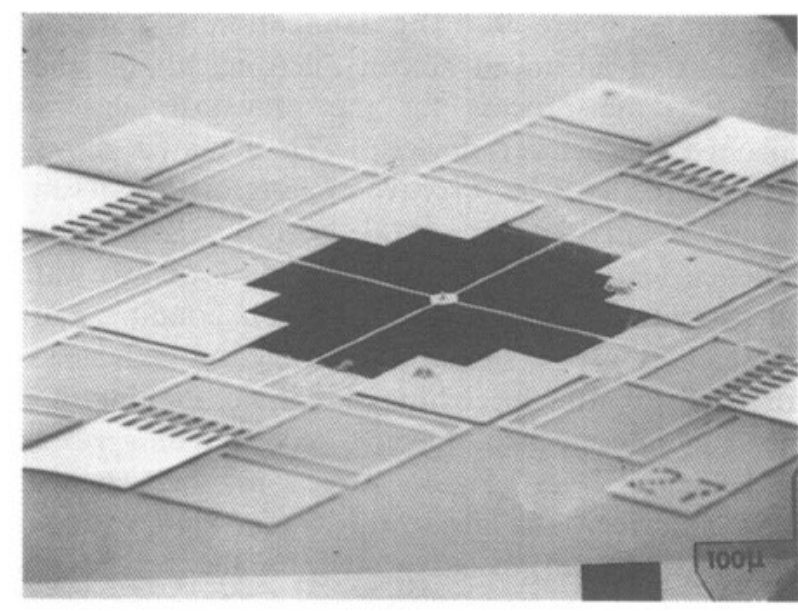

Figure 10. SEM micrograph overview of the micromachined single-crystalline silicon $x y$ nanoscanner.

been operated, representing an interesting class of miniaturized surface-scanned devices. Single-crystal silicon has proved to be most promising for the integration of a sensing tip and a scanning unit. A first prototype of such a microfabricated $x y$ nanoactuator with vertically protruding tip on an overhanging $x y$ comb drive actuator has been shown. The presented devices can be successfully integrated into an 'on-chip SFM'. The continuous advances in the microfabrication of silicon will enable the further integration of these tools into a fully micromachined MEMS for nanoscience.

\section{Acknowledgments}

The authors are indebted to $\mathrm{R} A$ Buser for initiating this project. We would like to acknowledge $M$ Binggeli, $R$ Christoph, Ph Renaud and J M Moret from the Swiss Center for Electronics and Microtechnology (CSEM) Inc as well as the teams of Professor Güntherodt, University of Basel, and Dr H Rohrer, IBM Rüschlikon, for their continual interest in this work and valuable discussions. The excellent technical assistance provided

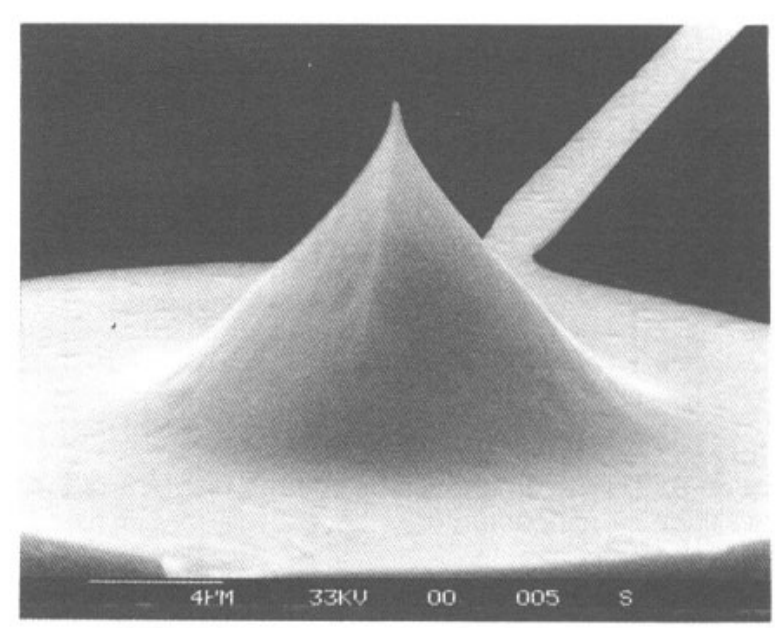

Figure 11. Magnified SEM micrograph showing details of the integrated tip on the center stage.

by S Jeanneret, P A Clerc and S Pochon from the IMT and the technical staff from CSEM is also gratefully acknowledged. This project was financially supported by the Swiss National Science Foundation and the Swiss Foundation for Microtechnology Research.

\section{References}

[1] Binnig G, Rohrer H, Gerber Ch and Weibel E 1982 Surface studies by scanning tunneling microscopy Phys. Rev. Lett. 4957

[2] Binnig G, Quate C F and Gerber Ch 1986 Atomic force microscopy Phys. Rev. Lett. $\mathbf{5 6} 930$

[3] See e.g., Rugar D and Hansma P 1990 Atomic force microscopy Physics Today 2; Sanid D 1991 Scanning Force Microscopy (Oxford: Oxford University Press); Güntherodt H-J and Wiesendanger R (ed) 1992 Scanning Tunneling Microscopy, Further Applications and Related Techniques (Springer Series in Surface Sciences 28) (Berlin: Springer)

[4] Hosaka S 1993 personal communication

[5] See e.g., Hosaka S, Koyanagi H and Kikukawa A 1993 Nanometer recording on graphite and $\mathrm{Si}$ substrates using an atomic force microscope in air Japan $J$. Appl. Phys. 32 L464-7 
[6] Eigler D M and Schweizer E K 1990 Positioning single atoms with a scanning tunneling microscope Nature $344524-6$

[7] Quate C F 1991 Switch to atom control Nature 352571

[8] Morishita H and Hatamura Y 1993 Development of a ultra precise manipulator system for future nanotechnology 1st Workshop on Micro Robotics and Systems (Karlsruhe, 1993)

[9] Petersen K E 1978 Dynamic micromechanics on silicon: techniques and devices IEEE Trans. Electron Devices ED-25 1241-50

[10] MacDonald N C 1993 Nanomechanisms and tips for microinstruments Digest Tech. Papers Transducers 93 (Yokohama) 8-12

[11] Rangelow I W 1992 Nano-resolution tri-level process by downstream-microwave rf-biased etching Microelectron. Eng. 17 349-52

[12] Griffith J E and Grigg D A 1993 Dimensional metrology with scanning probe microscopes $A p p l$. Phys. Rev. at press

[13] Ravi T S, Marcus R B and Liu D 1991 Oxidation sharpening of silicon tips J. Vac. Sci. Technol. B 9 2733-7

[14] Akamine S and Quate C F 1992 Low temperature thermal oxidation sharpening of microcast tips $\mathrm{J}$. Vac. Sci. Technol. B 10 2307-10

[15] See, e.g., Yamadi $M$, Miwa T, Yoshimura $H$ and Nagayama K 1992 Efficient microtip fabrication with carbon coating and electron beam deposition for atomic force microscopy $J$. Vac. Sci Technol. B 10 2447-50

[16] See, e.g., Ishitani T, Ohnishi T and Kawanami Y 1990 Micromachining and device transplantation using focused ion beam Japan. J. Appl Phys. 29 2283-7

[17] Albrecht T, Akamine S, Carver T E and Quate C F 1990 Microfabrication of cantilever styli for the atomic force microscope J. Vac Sci. Technol. A 8 3386

[18] Wolter O, Bayer Th and Greschner J 1991 Micromachined silicon sensors for scanning force microscopy J. Vac. Sci Technol. B 91353

[19] Farooqui M M and Evans A G R 1993 Silicon sensors with integral tips for atomic force microscopy: a novel single-mask fabrication process $J$. Micromech Microeng. 3 3-12

[20] Tortonese M, Barrett R C and Quate C F 1993 Atomic resolution with an atomic force microscope using piezoresistive detection Appl. Phys. Lett. 6222

[21] Itoh T and Suga T 1993 Piezoelectric force sensor for scanning force microscopy Digest Tech Papers, Transducers 93 (Yokohama) p 610

[22] Akamine S, Albrecht T R, Zdeblick M J and Quate C F 1990 A planar process for microfabrication of a scanning tunneling microscope Sensors Actuators A 21-23 964-70

[23] Yao J J, Arney S C and MacDonald N C Fabrication of high frequency two-dimensional nanoactuators for scanned probe devices J. Microelectromech. Syst. 1 14-22

[24] Kobayashi D, Hirano T, Furuhata T and Fujita H 1992 An integrated lateral tunneling unit Digest MEMS Workshop (Travemünde, 1992) p 214

[25] Hirano T, Furuhata T, Gabriel K J and Fujita H 1992 Design, fabrication and operation of submicrometer gap comb-drive microactuators $J$. Microelectromech. Syst. 1 52-9

[26] Jaecklin V P, Linder C, de Rooij N F, Moret J-M, Bischof R and Rudolf F 1992 Novel polysilicon comb-actuators for xy-stages Tech. Digest IEEE Micro Electro Mechanical Systems Workshop (Travemünde 1992) pp 147-9

[27] Kim Ch-J, Pisano A P and Muller R S 1992
Silicon-processed overhanging microgripper $J$. Microelectromech. Syst. $131-6$

[28] Bocko M F and Stephenson K A 1991 Tunneling transducers: quantum limited displacement monitors at the nanometer scale J. Vac. Sci. TechnoL B 9 1363-6

[29] Rockstad H K, Kenny T W, Reynolds J K, Kaiser W J and Gabrielson T B 1993 A miniature high-sensitivity broad-band accelerometer based on electron tunneling transducers Tech Digest Transducers 93 (Yokohama) pp 835-9

[30] Linder C, Tschan T and de Rooij N F 1992 Deep dry etching of silicon-a novel micromachining tool Sensors Materials (Tokyo 1992) pp 311-24

[31] Buser R A, Brugger J and de Rooij N F 1991 Micromachined silicon cantilevers and tips for bidirectional force microscopy STM Conf. (Interlaken 1991) Utramicroscopy 42-4 1476-80; Brugger J, Buser R A and de Rooij N F 1992 Microfabricated silicon cantilevers and tips for the scanning force microscope Sensors Actuators A 34 193-200; Buser $R$, Brugger $J$ and Linder $C$ (Appls \& Invs) British Technology Group Ltd (Appl) 1992 Microprobe for surface scanning microscopes Patent PCT/GB92/01060

[32] Binnig G, Dürig U, Gimzewski J K, Pohl D and Rohrer H Atomic force sensor head Eur. Patent Application EP-A 0290648

[33] Kong L C, Orr B G and Wise K D 1990 A micromachined silicon scan tip for an atomic force microscope IEEE Solid-State Sensor and Actuator Workshop (Hilton Head Island, 1990) (New York: IEEE) pp 28-31

[34] Göddenhenrich T, Lemke $H$, Hartmann $U$ and Heiden C 1990 Force microscopy with capacitive displacement detection J. Vac. Sci. Technol. A 8 383-7

[35] Neubauer $G$, Cohen S R, McClelland G M, Horne $D$ and Mate C M 1990 Force microscopy with a bidirectional capacitance sensor Rev. Sci. Instrum. 61 2296-308

[36] Brugger J, Buser R A and de Rooij N F 1992 Micromachined atomic force microprobe with integrated capacitive read-out J. Micromech. Microeng. 2 218-20; Brugger J, Blanc N, Renaud $\mathrm{Ph}$ and de Rooij N F 1993 Capacitive AFM microlever with combined integrated sensor/actuator functions Digest Tech Papers, Transducers 93 (Tokohama) p 1044

[37] Linder C 1993 Electromechanical polysilicon structures and micromachining processes for sensor and actuator applications $P h D$ Thesis University of Neuchâtel

[38] Nathanson $H$ C, Newell W E, Wickstrom R A and Davis Jr J R 1967 The resonant gate transistor IEEE Trans. Electron Devices ED-14 117-33

[39] Buser R A and de Rooij N F 1990 Very high Q-factor resonators in monocrystalline silicon Sensors Actuators A 21-23 323-7

[40] Burns D W and Guckel H 1990 Thin films for micromechanical sensors $J$. Vac. Sci. Technol. A 8 3606-13

[41] Linder C, Paratte L, Gretillat M-A, Jaecklin V P and de Rooij N F Surface micromachining $J$. Micromech. Microeng. 2 122-32

[42] Jaecklin V P, Linder C, de Rooij N F and Moret J M Micromechanical comb actuators with low driving voltage J. Micromech. Microeng. 2 250-5

[43] Brugger J, Jaecklin V P, Indermühle P F, Linder C and de Rooij N F 1993 Fabrication of an overhanging $x y$-microactuator with integrated tip for scanned surface profiling Late News Paper Transducers 93 (Yokohama) pp \&-9 